\title{
Percutaneous transhepatic obliteration and percutaneous transhepatic sclerotherapy for intractable hepatic encephalopathy and gastric varices improves the hepatic function reserve
}

\author{
TORU ISHIKAWA, MICHITAKA IMAI, MASAYOSHI KO, HIROKI SATO, YUJIRO NOZAWA, \\ TOMOE SANO, AKITO IWANAGA, KEIICHI SEKI, TERASU HONMA and TOSHIAKI YOSHIDA \\ Department of Gastroenterology and Hepatology, Saiseikai Niigata Daini Hospital, Niigata 950-1104, Japan
}

Received September 2, 2016; Accepted October 27, 2016

DOI: $10.3892 /$ br.2016.811

\begin{abstract}
Percutaneous transhepatic obliteration (PTO) and percutaneous transhepatic sclerotherapy (PTS) are widely performed as an emergency measure in cases of variceal hemorrhage and intractable hepatic encephalopathy. The PTO/PTS technique is capable of directly blocking the blood supply in cases in which balloon-occluded retrograde transvenous obliteration (B-RTO) is not effective, or in cases with complicated collateral flow. Although PTO/PTS is not currently the first choice due to the invasiveness of transhepatic puncture, this procedure can modify the blood flow in an antegrade manner. The present study examined the changes in hepatic function reserve following PTO/PTS for intractable hepatic encephalopathy and/or gastric varices. In total, the study included 37 patients (mean age, $61.75 \pm 12.77$ years; age range, 32-88 years; male to female ratio, 23:14) with a variety of gastrorenal shunts, or B-RTO-intractable hepatic encephalopathy and gastric varices without gastrorenal shunts. The patients underwent PTO/PTS by embolizing a microcoil or injection of a sclerosing agent $(5 \%$ ethanolamine oleate iopamidol). Alterations in hepatic function reserve prior to and following the procedure were compared. The patients were treated for hepatic encephalopathy in 11 patients, gastric varices in 19 patients, and both conditions in 7 patients. The results indicated that the blood ammonia level improved from $135.76 \pm 75.23 \mathrm{mg} / \mathrm{dl}$ to $88.00 \pm 42.16$ and $61.81 \pm 33.75 \mathrm{mg} / \mathrm{dl}$ at 3 and 6 months after therapy, respectively. In addition, the Child-Pugh score improved from $8.48 \pm 2.01$ prior to therapy to $7.70 \pm 1.84$ and $7.22 \pm 2.01$ at 3 and 6 months after the procedure,
\end{abstract}

Correspondence to: Dr Toru Ishikawa, Department of Gastroenterology and Hepatology, Saiseikai Niigata Daini Hospital, 280-7 Teraji, Niigata 950-1104, Japan

E-mail: toruishi@ngt.saiseikai.or.jp

Key words: percutaneous transhepatic obliteration, percutaneous transhepatic sclerotherapy, portal hypertension, hepatic function reserve, gastric varices, hepatic encephalopathy respectively. Although there was a concern that PTO/PTS may cause complications due to an increase in portal venous pressure (PVP) arising from shunt occlusion, no severe complications were observed. In conclusion, for patients with various gastrorenal shunts or those with B-RTO-intractable hepatic encephalopathy and gastric varices without gastrorenal shunts, PTO/PTS can improve the antegrade blood flow to the liver, as demonstrated by improvement in the hepatic function reserve.

\section{Introduction}

Chronic liver disease causes portal hypertension through progression from hepatitis to cirrhosis. In portal hypertension, a variety of adverse effects are induced by hemodynamic fluctuations, including gastroesophageal variceal and portosystemic shunt formation (1). Progression of portal hypertension further reduces afferent blood flow to the liver, leading to a decrease in hepatic function reserve (2). Therapies for portal hypertension are widely categorized into portal pressure reduction therapy and shunt occlusion therapy. A transjugular intrahepatic portosystemic shunt $(3,4)$ and partial splenic embolization (5) are examples of pressure reduction therapy, whereas shunt occlusion involves percutaneous transhepatic obliteration (PTO) (6), percutaneous transhepatic sclerotherapy (PTS) $(7,8)$, balloon-occluded retrograde transvenous obliteration (B-RTO) (9-11), and combined BRTO and PTO therapy (12). BRTO is a procedure that has become common practice in Japan, and is popular in South Korea for the management of gastric varices $(9,13,14)$. However, certain cases of gastric varices without catheterizable main draining veins cannot be treated by BRTO. PTS has recently been introduced for the treatment of gastric varices without gastrorenal shunts (15) with satisfactory treatment results reported for the patients. In these cases, it is necessary to adopt an antegrade approach by PTO/PTS. Although PTO/PTS is not currently the first choice, due to the invasiveness of transhepatic puncture, this procedure modifies the blood flow in an antegrade manner. The present study examined whether hepatic function reserve improves following PTO/PTS for hepatic encephalopathy and intractable gastroesophageal varices due to portosystemic shunting caused by portal hypertension. 


\section{Materials and methods}

Study participants and treatment. Fluctuations in hepatic function reserve were investigated in 37 patients who underwent PTO/PTS with a variety of gastrorenal shunts, or B-RTO-intractable hepatic encephalopathy and gastric varices without gastrorenal shunts at Saiseikai Niigata Daini Hospital (Niigata, Japan) between January 2010 and March 2016. All patients were diagnosed with variceal hemorrhage and intractable hepatic encephalopathy. Written informed consent was obtained from all patients, and the Ethics Committee of Saiseikai Niigata Daini Hospital approved this study, which was conducted in accordance with the 1975 Declaration of Helsinki.

Procedure. For intractable hepatic encephalopathy, medical treatment eliminates ammonia from the gastrointestinal tract; for example, lactulose is administered to reduce serum ammonia levels. Prior to PTO/PTS, ultrasonography was performed for all patients in order to determine the best access route into the portal venous system. The procedure was performed on inpatients under conscious sedation using a combination of $25 \mathrm{mg}$ intramuscular hydroxyzine (Atarax P; Pfizer Japan Inc., Tokyo, Japan) and $15 \mathrm{mg}$ pentazocine (Pentagin; Daiichi Sankyo Co., Ltd., Tokyo, Japan) administered $15 \mathrm{~min}$ before treatment. After $10 \mathrm{ml}$ of $1 \%$ lidocaine (Xylocaine; AstraZeneca K.K., Osaka, Japan) was injected into the peritoneum along the puncture line, percutaneous transhepatic puncture of the intrahepatic branch of the portal vein was performed using an 18-gauge needle under sonographic guidance. A 5-French gauge sheath catheter was then introduced into the portal vein. Direct portography was performed to identify the feeding and draining veins of the gastric varices or shunt veins. The gastric varices or shunt veins often had multiple feeding veins, and a coaxial catheter was inserted into these feeding veins while avoiding the main feeding vein. The feeding veins were embolized with microcoils or a sclerosing agent, namely $5 \%$ ethanolamine oleate iopamidol (Oldamin; Mochida Pharmaceutical, Tokyo, Japan). PTO is usually performed by placing metallic coils in the afferent veins to reduce blood flow. Hence, PTS is usually performed by injection of sclerosing agent in the afferent veins to reduce blood flow (16).

Measurements. Analyses were performed to determine whether PTO/PTS improves hepatic functional reserve. Alterations in serum albumin, total bilirubin, prothrombin activity, platelet counts, blood ammonia level were observed during the follow-up period, and the Child-Pugh score (17) prior to PTO/PTS, and at 3 and 6 months after the procedure were compared. In addition, age, gender, etiology of liver cirrhosis and therapeutic purpose were observed. Statistical analysis. Variable data are expressed as the mean \pm standard deviation. Categorical variables were compared using $\chi^{2}$ test or Fisher's exact test, where appropriate. Continuous variables were compared using the independent sample Student's t-test or one-way repeated analysis of variance. Values of $\mathrm{P}<0.05$ were considered to indicate statistically significant differences. Statistical processing was performed using StatView version 5.0 software (SAS Institute, Cary, NC, USA).
Table I. Patient characteristics.

\begin{tabular}{lc}
\hline Characteristic & Value \\
\hline Age range, years & $32-88$ \\
Mean age \pm standard deviation, years & $61.75 \pm 12.77$ \\
Gender (male:female) & $23: 14$ \\
Etiology of liver disease & \\
HBV & 2 \\
HCV & 5 \\
HBV and HCV & 2 \\
Alcohol & 16 \\
IPH & 3 \\
NASH & 4 \\
PBC & 4 \\
Wilson's disease & 1 \\
Patient condition & \\
Gastric varices & 19 \\
Hepatic encephalopathy & 11 \\
Gastric varices and hepatic encephalopathy & 17 \\
Child-Pugh score & \\
A & 8 \\
B & 15 \\
C & 14 \\
\hline
\end{tabular}

$\mathrm{HBV}$, hepatitis B virus; $\mathrm{HCV}$, hepatitis C virus; IPH, idiopathic portal hypertension; NASH, non-alcoholic steatohepatitis; $\mathrm{PBC}$, primary biliary cirrhosis.

\section{Results}

Patient characteristics. The clinical characteristics of the 37 patients included in the present study are shown in Table I. The mean age was $61.76 \pm 12.77$ years (age range, $32-88$ years), and the male to female ratio was 23:14. The reasons for treatment included hepatic encephalopathy in 11 patients (29.7\%), gastric varices in 19 patients (51.3\%), and both conditions in 7 patients $(18.9 \%)$. In total, $8(21.6 \%), 15(40.5 \%)$ and $14(37.8 \%)$ patients were scored before treatment as Child-Pugh grade A, B and C, respectively. The etiology of liver cirrhosis combined with variceal hemorrhage and intractable hepatic encephalopathy were as follows: Hepatitis B viral infection, hepatitis $\mathrm{C}$ viral infection, or infection with both viruses in 2 (5.4\%), $5(13.5 \%)$ and $2(5.4 \%)$ patients, excessive alcohol consumption in $16(43.2 \%)$, idiopathic portal hypertension in $3(8.1 \%)$, nonalcoholic steatohepatitis in $4(10.8 \%)$, primary biliary cirrhosis in 4 (10.8\%), and Wilson's disease in $1(2.7 \%)$ patient, respectively.

Patient outcome. The PTO/PTS procedure was successful in all cases. The postoperative portal venous pressure (PVP) was $34.75 \pm 5.94 \mathrm{~cm} \mathrm{H}_{2} \mathrm{O}\left(24.5-42.0 \mathrm{~cm} \mathrm{H}_{2} \mathrm{O}\right)$ immediately following surgery. No severe complications due to PTO/PTS were observed. However, a recurrent ectopic gastric varix was found in 1 patient who underwent PTO/PTS for gastric varices. 
Table II. Sequential changes prior to and following PTO/PTS.

\begin{tabular}{lcccc}
\hline Category & Pre-PTO/PTS & 3 months after PTO/PTS & 6 months after PTO/PTS & P-value \\
\hline Serum albumin $(\mathrm{g} / \mathrm{dl})$ & $2.99 \pm 0.57$ & $2.98 \pm 0.51$ & $3.13 \pm 0.60$ & $\mathrm{P}=0.0717$ \\
Total bilirubin $(\mathrm{mg} / \mathrm{dl})$ & $1.34 \pm 1.01$ & $1.29 \pm 0.89$ & $7.23 \pm 0.82$ & $\mathrm{P}=0.5775$ \\
Prothrombin activity $(\%)$ & $74.02 \pm 17.61$ & $71.48 \pm 19.88$ & $16.27 \pm 22.43$ & $\mathrm{P}=0.0435$ \\
Platelet count $\left(\mathrm{x} 10^{4} / \mathrm{ml}\right)$ & $12.80 \pm 7.29$ & $14.18 \pm 7.12$ & $14.95 \pm 7.43$ & $\mathrm{P}=0.0209$ \\
Blood $\mathrm{NH}_{3}(\mu \mathrm{g} / \mathrm{dl})$ & $135.75 \pm 75.23$ & $88.80 \pm 42.16$ & $61.81 \pm 33.75$ & $\mathrm{P}<0.001$ \\
\hline
\end{tabular}

PTO/PTS, percutaneous transhepatic obliteration/percutaneous transhepatic sclerotherapy.

Measurements. During the treatment course, serum albumin level deteriorated from $2.99 \pm 0.57 \mathrm{~g} / \mathrm{dl}$ (range, 2.10-4.30) to $2.98 \pm 0.51 \mathrm{~g} / \mathrm{dl}$ (range, 1.90-4.10) at 3 months after PTO/PTS; however, this level improved to $3.13 \pm 0.60 \mathrm{~g} / \mathrm{dl}$ (range, 2.70-4.30) at 6 months after PTO/PTS (Table II). Prothrombin activity also deteriorated from $74.02 \pm 17.61 \%$ (range, 46.00-94.40) to $71.48 \pm 19.88 \%$ (range, $25.00-90.70$ ) at 3 months after PTO/PTS, but improved to $76.27 \pm 22.43 \%$ (range, 42.00-116.30) at 6 months after PTO/PTS. By contract, the total bilirubin activity was not significantly changed prior to and following PTO/PTS (Table II).

The platelet count significantly improved from $12.80 \pm 7.29 \times 10^{4} / \mu 1$ (range, $4.10-32.50$ ) to $14.18 \pm 7.11 \times 10^{4} / \mu 1$ (range, 5.00-30.90) and 14.95 $\pm 7.43 \times 10^{4} / \mu 1$ (range, 5.90-36.50) at 3 and 6 months after therapy, respectively $(\mathrm{P}=0.0209)$. Blood ammonia levels also improved from $135.75 \pm 75.23 \mu \mathrm{g} / \mathrm{dl}$ (range, $38.00-410.00$ ) to $88.00 \pm 42.16 \mu \mathrm{g} / \mathrm{dl}$ (range, 23.00-205.00) and $61.81 \pm 33.75 \mu \mathrm{g} / \mathrm{dl}$ (range, $15.00-191.00$ ) at 3 and 6 months after therapy, respectively (Table II). Furthermore, the Child-Pugh score improved from 8.48 \pm 2.01 (range, 5.0-13.0) prior to therapy to $7.70 \pm 1.84$ (range, 5.0-12.0) and 7.22 \pm 2.01 (range, 5.0-11.0) at 3 and 6 months after therapy, respectively (Fig. 1; $\mathrm{P}<0.05)$.

\section{Discussion}

An increase in shunt blood flow due to portal hypertension caused by progression of cirrhosis leads to an increase in blood flow bypassing the liver, resulting in hepatic encephalopathy (also known as shunt encephalopathy) (18). In various cases, symptoms are stabilized by conservative therapy, including administration of a branched-chain amino acid preparation, such as lactulose. However, even if the state of consciousness is improved temporarily by conservative therapy, it is not uncommon for shunt blood flow to increase excessively, resulting in persistent hyperammonemia or facilitating a change in mental status.

The presence of a portosystemic shunt causes hepatic encephalopathy due to toxic substances, including intestine-derived ammonia, flowing directly into the systemic circulation and bypassing the liver (19). Furthermore, collateral flow induces intractable gastroesophageal varices (20). In addition to these pathologies, a further decrease in hepatic function reserve is a concern due to the reduction in portal blood flow as a result of the presence of a portosystemic shunt. Methods of shunt occlusion include surgical treatment and

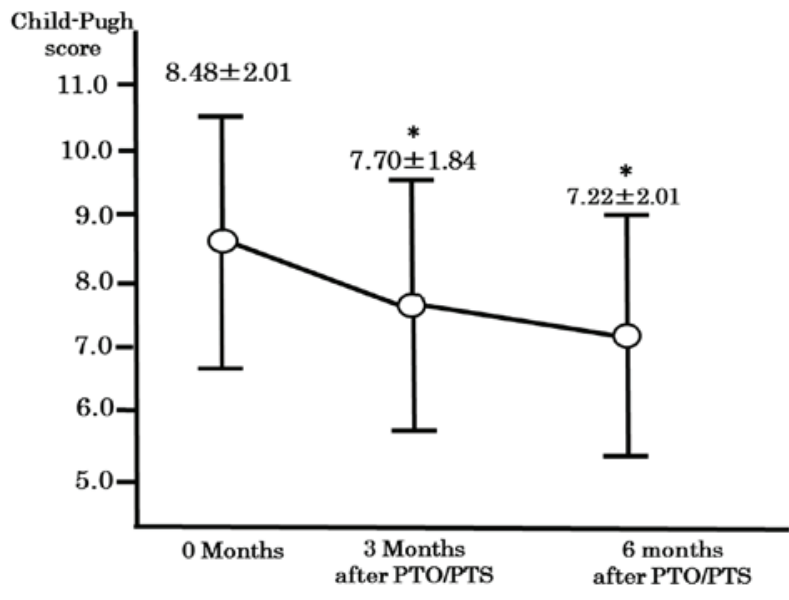

Figure 1. Comparison of the changes in Child-Pugh score prior to and following treatment with PTO/PTS at different time-points. The Child-Pugh score improved from $8.48 \pm 2.01$ (range, 5.0-13.0) prior to therapy to $7.70 \pm 1.84$ (range, 5.0-12.0) and 7.22 2.01 (range, 5.0-11.0) at 3 and 6 months after therapy, respectively. ${ }^{*} \mathrm{P}<0.05$ vs. before PTO/PTS. PTO/PTS, percutaneous transhepatic obliteration/percutaneous transhepatic sclerotherapy.

interventional radiology (IVR). When based on the direction of blood flow, IVR can be classified into two types, one including B-RTO (9-11) and transjugular retrograde obliteration for gastric varices $(21,22)$, which are retrograde embolizations, and the other including transileocolic venous obliteration (23) and PTO (6), which are antegrade embolizations.

PTO, a therapy initially devised by Lunderquist and Vang in 1974, had been widely performed as an emergency measure in cases of variceal hemorrhage (6). However, the development of a new blood supply route following occlusion occasionally causes recurrence of varices or hemorrhage. Therefore, PTO has the disadvantage of being relatively invasive due to transhepatic puncture. However, it enables understanding of hemodynamics relatively easily in angiography, as it is an antegrade embolization technique. The PTS technique has been recommended as a good option in such cases $(16,24)$. Ninoi et al (16) performed PTS in patients with gastric varices without a gastrorenal shunt, in patients with gastric varices with a gastrorenal shunt and gastrocaval shunts, and in patients with gastric varices not treatable by BRTO. The current study described coil embolization of the afferent veins and the use of $5 \%$ ethanolamine oleate with iopamidol as the sclerosant.

PTS is usually performed by placing metallic coils in the afferent veins to reduce blood flow into the gastric varix, after 
which a sclerosing agent is injected in the antegrade direction into the gastric varix $(16,17)$. When there are multiple afferent veins, this procedure may need to be performed for all these veins.

The present study examined whether improvement in intrahepatic blood flow with PTS/PTO leads to an improvement in hepatic function reserve. No severe complications due to PTO/PTS were observed. However, a recurrent ectopic gastric varix was found in 1 patient who underwent PTO/PTS for gastric varices.

The improvement in the Child-Pugh score observed in the patients of the current study indicated that PTO/PTS contributes to the improvement in hepatic function reserve. In addition, although there was a concern that PTO/PTS may cause certain complications due to an increase in PVP arising from shunt occlusion, no severe complications were observed.

In conclusion, depending on the objective of obliteration and the hepatic hemodynamics in each case, PTO/PTS performed with caution should improve or maintain hepatic function reserve. Therefore, PTO/PTS is useful as a therapeutic strategy for portal hypertension in patients with various gastrorenal shunts, or B-RTO-intractable hepatic encephalopathy and gastric varices without gastrorenal shunts.

\section{References}

1. Palmer ED: On correlations between portal venous pressure and the size and extent of esophageal varices in portal cirrhosis. Ann Surg 138: 741-744, 1953.

2. Palmer ED, Sborov VM and Jahnke EJ Jr: Effects of surgical treatment of esophageal varices on the portal venous pressure and hepatic function: Preliminary observations. Gastroenterology 24: 10-15, 1953.

3. Blei AT: TIPS and variceal bleeding. Ann Intern Med 117: 266, 1992.

4. McCormick PA, Dick R and Burroughs AK: Review article: The transjugular intrahepatic portosystemic shunt (TIPS) in the treatment of portal hypertension. Aliment Pharmacol Ther 8: 273-282, 1994.

5. Spigos DG, Jonasson O, Mozes M and Capek V: Partial splenic embolization in the treatment of hypersplenism. AJR Am J Roentgenol 132: 777-782, 1979.

6. Lunderquist A and Vang J: Transhepatic catheterization and obliteration of the coronary vein in patients with portal hypertension and esophageal varices. N Engl J Med 291: 646-649, 1974.

7. Okuda K, Kimura K and Takayasu K: Percutaneous transhepatic portography and sclerotherapy. Semin Liver Dis 2: 57-66, 1982.

8. Yoshimatsu R, Yamagami T, Miura H and Okuda K: Percutaneous transhepatic sclerotherapy with embolization of the drainage vein for a gastric varix. Acta Radiol Short Rep 3: 2047981614530285 , 2014.

9. Kanagawa H, Mima S, Kouyama H, Gotoh K, Uchida T and Okuda K: Treatment of gastric fundal varices by balloon-occluded retrograde transvenous obliteration. J Gastroenterol Hepatol 11: 51-58, 1996.
10. Hirota S, Matsumoto S, Tomita M, Sako M and Kono M: Retrograde transvenous obliteration of gastric varices. Radiology 211: 349-356, 1999.

11. Kobayashi K, Maruyama H, Kiyono S, Sekimoto T, Kondo T, Shimada T, Takahashi M, Okugawa $\mathrm{H}$ and Yokosuka O: Portal response related to shunt occlusion by balloon-occluded retrograde transvenous obliteration may determine the prognosis of cirrhosis. Hepatol Res: Mar 2, 2016 (Epub ahead of print).

12. Fukatsu H, Kawamoto H, Harada R, Tsutsumi K, Fujii M, Kurihara N, Ogawa T, Ishida E, Okamoto Y, Okada H, et al: Gastric fundal varices with an exposed microcoil after the combined BRTO and PTO therapy. Endoscopy 39 (Suppl 1): E247-E248, 2007.

13. Kiyosue H, Mori H, Matsumoto S, Yamada Y, Hori Y and Okino Y: Transcatheter obliteration of gastric varices. Part 1. Anatomic classification. Radiographics 23: 911-920, 2003.

14. Ibukuro K, Sugihara T, Tanaka R, Fukuda H, Abe S, Tobe K, Tateishi $\mathrm{R}$ and Tagawa K: Balloon-occluded retrograde transvenous obliteration (BRTO) for a direct shunt between the inferior mesenteric vein and the inferior vena cava in a patient with hepatic encephalopathy. J Vasc Interv Radiol 18: 121-125, 2007.

15. Kiyosue H, Matsumoto S, Yamada Y, Hori Y, Okino Y, Okahara $\mathrm{M}$ and Mori H: Transportal intravariceal sclerotherapy with N-butyl-2-cyanoacrylate for gastric varices. J Vasc Interv Radiol 15: 505-509, 2004.

16. Ninoi T, Nakamura K, Kaminou T, Nishida N, Sakai Y, Kitayama T, Hamuro M, Yamada R, Arakawa T and Inoue Y: TIPS versus transcatheter sclerotherapy for gastric varices. AJR Am J Roentgenol 183: 369-376, 2004.

17. Pugh RN, Murray-Lyon IM, Dawson JL, Pietroni MC and Williams R: Transection of the oesophagus for bleeding oesophageal varices. Br J Surg 60: 646-649, 1973.

18. Sherlock S (ed): Hepatic Cirrhosis. In: Diseases of the Liver and Biliary System. 8th edition. Blackwell Scientific Publications, Oxford, pp410-424, 1989.

19. Prakash R and Mullen KD: Mechanisms, diagnosis and management of hepatic encephalopathy. Nat Rev Gastroenterol Hepatol 7: 515-525, 2010

20. Uchiyama F, Murata S, Onozawa S, Nakazawa K, Sugihara F, Yasui D, Narahara Y, Uchida E, Amano Y and Kumita S: Management of gastric varices unsuccessfully treated by balloon-occluded retrograde transvenous obliteration: Long-term follow-up and outcomes. Sci World J 2013: 498535, 2013.

21. Chikamori F, Shibuya S, Takase Y, Ozaki A and Fukao K: Transjugular retrograde obliteration for gastric varices. Abdom Imaging 21: 299-303, 1996.

22. Chikamori F, Kuniyoshi N, Shibuya S and Takase Y: Transjugular retrograde obliteration for chronic portosystemic encephalopathy. Abdom Imaging 25: 567-571, 2000.

23. Ota K, Okazaki M, Higashihara H, Kokawa H, Shirai Z, Anan A, Kitamura Y and Shijo H: Combination of transileocolic vein obliteration and balloon-occluded retrograde transvenous obliteration is effective for ruptured duodenal varices. J Gastroenterol 34: 694-699, 1999.

24. Kameda N, Higuchi K, Shiba M, Kadouchi K, Machida H, Okazaki $\mathrm{H}$, Tanigawa $\mathrm{T}$, Watanabe $\mathrm{T}$, Tominaga $\mathrm{K}$, Fujiwara Y, et al: Management of gastric fundal varices without gastro-renal shunt in 15 patients. World J Gastroenterol 14: 448-453, 2008 